\title{
The value of magnetic resonance imaging in the diagnosis of penile fracture
}

\author{
Ibrahim Guler ${ }^{1}$, Kemal Ödev ${ }^{2}$, Havva Kalkan ${ }^{2}$, Cihan Simsek ${ }^{2}$, Suat Keskin ${ }^{2}$, Mehmet Kilinç ${ }^{3}$ \\ ${ }^{1}$ Department of Radiology, Konya Research and Education Hospital, Konya, Turkey; ${ }^{2}$ Department of \\ Radiology and ${ }^{3}$ Department of Urology, Necmettin Erbakan University Meram School of Medicine, Konya, \\ Turkey
}

\section{ABSTRACT}

Purpose: We studied the use of magnetic resonance imaging in the diagnosis of penile fracture.

Materials and Methods: Between 1997 and 2012, fifteen patients (age range 17-48 years, mean age 37 years) with suspected penile fracture underwent MRI examinations. Ten patients were injured during sexual intercourse, whereas four patients were traumatized by non-physiological bending of the penis during self manupilation, one patient was traumatized falling from the bed. Investigations were performed with $1.5 \mathrm{~T}$ MR unit. With the patient in the supine position, the penis was taped against the abdominal wall and surface coil was placed on the penis. All patients were studied with axial, coronal, sagittal precontrast and postcontrast T1-weighted TSE(TR/TE:538/13 msn) and T2-weighted TSE(5290/110 msn) sequences. All patient underwent surgical exploration. The follow-up ranged from 3 months to 72 months. Clinically all patients showed normal healing process without complications. In 11 patients a shortening and thickening of tunica albuginea was observed. Three patients have post traumatic erectil disfunction.

Results: In all patient corpus cavernosum fractures were clearly depicted on a discontinuity of the low signal intensity of tunica albuginea. These findings were most evident on T1WI and also depicted on T2W sequences. Images obtained shortly after contrast medium administration showed considerable enhancement only in rupture site. Subcutaneous extratunical haematoma in all patients were also recognizable on T2 WI. MRI findings were confirmed at surgery.

Conclusions: Magnetic resonance imaging is of great value for the diagnosis of penile fracture. Furthermore this method is well suited for visualising the post-operative healing process

\section{ARTICLE INFO}

\section{Key words:}

Penis; diagnosis [Subheading]; Magnetic Resonance Spectroscopy; Penile Induration

Int Braz J Urol. 2015; 41: 325-8

Submitted for publication:

March 28, 2014

Accepted after revision:

July 28, 2014

\section{INTRODUCTION}

Penile fracture is a rare urological emergency situation, and is defined as disruption of the tunica albuginea (TA) and corpus cavernosum (CC). It occurs almost exclusively as a result of blunt trauma to the penis in the erect position, usually during coitus. Other mechanisms of injury are direct external blunt trauma, abnormal bending of the penis during manipulation, forceful manipulation and rolling over in bed during erection (1-4). Disruption of the tunica albuginea or associated urethra injury are indications for surgical repair, whereas other cases of blunt penile injury may be treated conservatively. In general, the patient's history and physical examination are su- 
fficient to reach a provisional diagnosis of a penile fracture. The role of magnetic resonance imaging (MRI) in imaging penile fractures is to ascertain the integrity of the tunica albuginea. In this study we describe MRI findings at 15 patients with acute penile fracture and its healing process.

\section{MATERIALS AND METHODS}

From 1997 to 2012, fifteen patients (age range 17-48 years, mean age 37 years) with suspected penile fracture underwent MRI examinations. Ten patients were injured during sexual intercourse, whereas four patients were traumatized by non-physiological bending of the penis during self manipulation, one patient was traumatized falling from the bed. Investigations were performed with 1.5T MR unit (Magnetom Symphony, Siemens, Erlangen Germany). With the patient in the supine position, the penis was taped against the abdominal wall and surface coil was placed on the penis. All patients were studied with axial, coronal, sagittal T2-weighted TSE (5290/110 msn) and precontrast and postcontrast T1-weighted TSE (TR/TE:538/13msn) sequences.

All patients underwent surgical operation and repair via a longitudinal incision. The follow-up ranged from 3 months to 72 months.

\section{RESULTS}

In all patients CC fractures were clearly depicted on a discontinuity of the low signal intensity of TA. These findings were most evident on T1-weighted images (Figures 1a, 2a, 3a and b and 4a ) These findings were also depicted on T2-weighted images (Figures $1 \mathrm{~b}$ and c, 2b, 3c and d and 4b). Images obtained shortly after contrast medium administration showed considerable enhance only in rupture site. Subcutaneous extratunical haematoma in all patients were also recognizable on T2-weighted images. They were much more significant at post-contrast T1-weighted images. On follow-up in 10 patients a shortening and thickening of TA were observed (Figures 1d and 2d). There were erectile difficulties in 2 patients and they did not complain of any voiding difficulties. In all patients MR imaging findings were confirmed at surgery.
Figure 1 - A 37-year-old patient with penile fracture of the left corpus cavernosum. (a) Sagittal T1-weighted image shows a rupture of the tunica albuginea (arrow) and a haematoma extending into the corpus cavernosum (asteriks). Coronal (b) and sagittal (c) T2-weighted images show also disruption of tunica albuginea (arrow) with surrounding haematoma in the distal penile shaft (asteriks). (d) One year later after surgical intervention the tunica albuginea at the rupture site regains its low signal intensity (arrow).

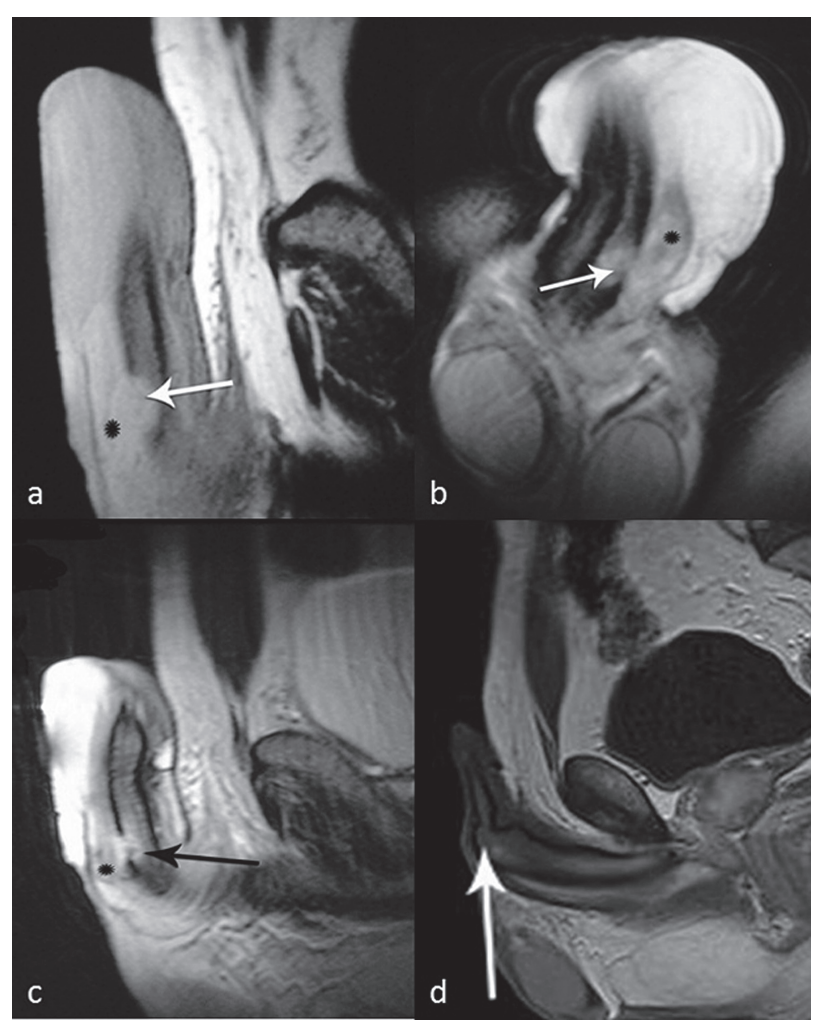

\section{DISCUSSION}

Penile fracture has a typical clinical presentation. Patients report hearing a snapping sound during the sexual act, followed by immediate pain and penile detumescence, in addition to the emergence of large edema, hematoma, and penile deformity (5). In general the diagnosis of penile fracture is easily established clinically.

The diagnosis is usually not difficult and often is based on history and physical examination. Patients with atypical clinical findings may require a common diagnostic procedure. Sonography could be also a useful imaging modality for the diagnosis of penile fracture because it is easy to perform, noninvasive, widely available and 
Figure 2 - A 30-year-old patient with penile fracture of the right corpus cavernosum. Sagittal T1-weighted (a) sagittal T2-weighted (b) and coronal T1-weighted (c) images show a disruption of the tunica albuginea (arrow) with surrounding haematoma in the penile midshaft (asteriks). (d) Two years later after surgical repair of the the tunica albuginea, T1weighted image shows a thickening of the rupture site (arrow).

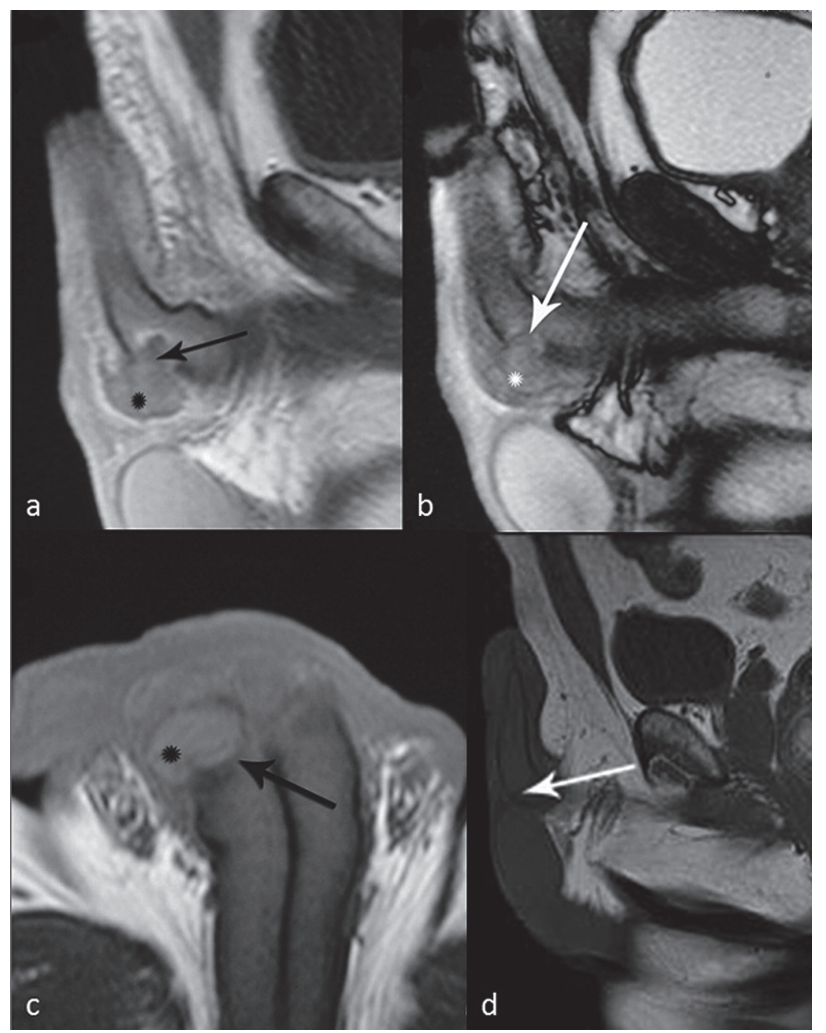

unexpensive (6). However this technique is operator dependent, and the major obstacle is a lack of tissue contrast (7). Other limitations of the sonography are that, edematous swelling of the penis and clots within the tear deteriorate the image contrast and can obscure the defect; furthermore, a rupture in the pendilous area cannot be visualised. Some authors advocate cavernosonography to delinate the corporal rupture, but this invasive and painful procedure may increase heamatoma and involves ionizing radiation with a risk of infection and sometimes a lack image contrast.

Because of MRI multiplanar capabilty and excellent tissue contrast, it can be useful as a diagnostic tool in the evaluation of patients with acute penile fracture (8).
Figure-3 - A 40-year-old patient with penile fracture of the right corpus cavernosum. Unenhanced (a) and enhanced (b) T1-weighted images fail to reveal the tunical tear, whereas axial (c) coronal (d) T2-weighted images identify the tunical tear (arrows) with surrounding haematoma of the right corpus cavernosum in the proximal penile (asteriks).

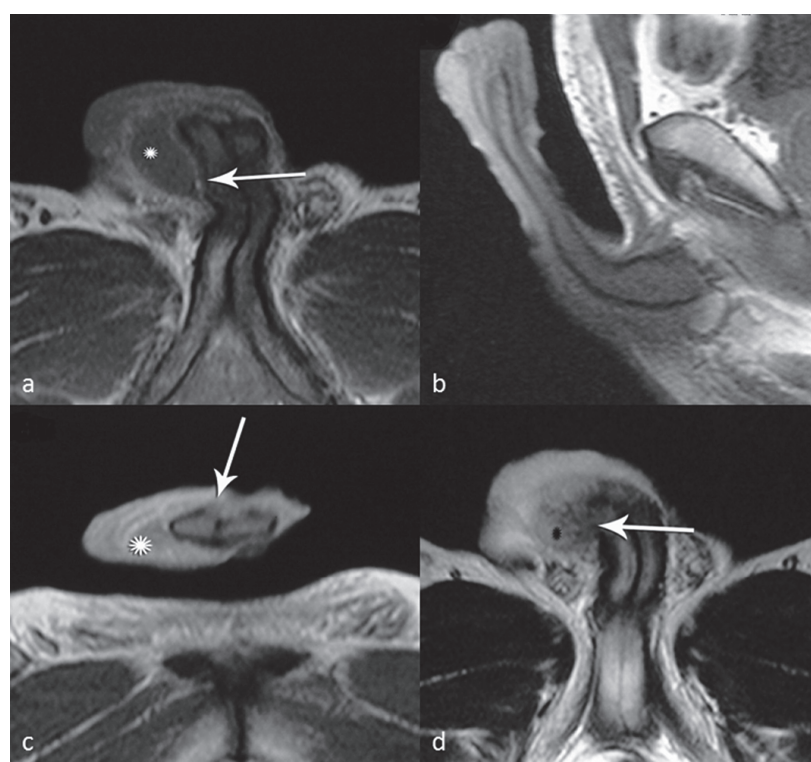

Figure 4 - A 30-year-old patient with penile fracture of the right corpus cavernosum. (a) Sagittal T1-weighted image shows a rupture of the tunica albuginea (arrow). (b) The lesion of the tunica albuginea and soft tissue haematoma surrounding the corpus cevarnosum can be detected on T2-weighted images (arrow). (c) Eight weeks later after surgery, the tunica albuginea in healing process showed heterogeneous signal on unenhanced T1-weighted image (arrow).

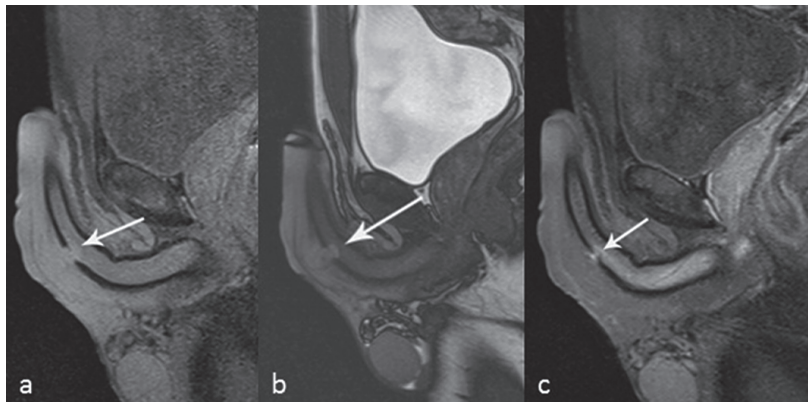

But MRI has also some limitations, such as higher cost than ultrasound, and readily not available everywhere. Especially in patients that ultrasound is not conclusive, MR imaging can accurately depict the presence, location and extent of tunical tear which manifest as discontunity of 
TA (9). Moreover, because TA is well demonstrated as a low-signal intensity structure on $\mathrm{T} 1$ and $\mathrm{T} 2$ weighted images, MR imaging is optimal for the evaluation of the integrity of this anatomic structure even in patients with severe pain and swelling of penis as well as hematomas that appear as high signal intensity on both $\mathrm{T} 1$ and T2-weighted images $(9,10)$. Moreover, associated injuries to adjacent structures (eg. corpus spongiosum, urethra) can also be demonstrated $(9,11)$. MR imaging can demonstrate an intact TA and the presence of intracavernosal and extratunical haematoma. MRI may also detect other pathologies that mimic penile fracture (12).

\section{CONCLUSIONS}

MR imaging is an excellent modality for evaluating patients with acute penile trauma. It can accurately demonstrate the integrity of the TA, as well as extent and location of tunical tear and of associated injuries to the CC and urethra.

\section{CONFLICT OF INTEREST}

None declared.

\section{REFERENCES}

1. Suzuki K, Shimizu N, Kurokawa K, Suzuki T, Yamanaka H. Fracture of the penis: magnetic resonance imaging of the rupture of the corpus cavernosum. Br J Urol.1995;76:803-4.

2. Boudghene F, Chhem R, Wallays C, Bigot JM. MR imaging in acute fracture of the penis. Urol Radiol. 1992;14:202-4.
3. Murray KS, Gilbert M, Ricci LR, Khare NR, Broghammer J. Penile fracture and magnetic resonance imaging. Int Braz $\mathrm{J}$ Urol. 2012;38:287-8.

4. Koifman L, Barros R, Júnior RA, Cavalcanti AG, Favorito LA. Penile fracture: diagnosis, treatment and outcomes of 150 patients. Urology. 2010;76:1488-92.

5. Eke N. Fracture of the penis. Br J Surg. 2002;89:555-65.

6. Bhatt S, Kocakoc E, Rubens DJ, Seftel AD, Dogra VS. Sonographic evaluation of penile trauma. J Ultrasound Med. 2005;24:993-1000; quiz 1001.

7. Choi MH, Kim B, Ryu JA, Lee SW, Lee KS. MR imaging of acute penile fracture. Radiographics. 2000;20:1397-405. Erratum in: Radiographics 2000;20:1818.

8. Yokogi H, Mizutami M, Ishibe T. Magnetic resonance imaging of a penile fracture. Acta Urol Belg. 1992;60:93-5.

9. Kirkham A. MRI of the penis. Br J Radiol. 2012;85:S86-93.

10. Fedel M, Venz S, Andreessen R, Sudhoff F, Loening SA. The value of magnetic resonance imaging in the diagnosis of suspected penile fracture with atypical clinical findings. J Urol. 1996;155:1924-7.

11. Uder M, Gohl D, Takahashi M, Derouet H, Defreyne L, Kramann B, et al. MRI of penile fracture: diagnosis and therapeutic follow-up. Eur Radiol. 2002;12:113-20.

12. Abolyosr A, Moneim AE, Abdelatif AM, Abdalla MA, Imam HM. The management of penile fracture based on clinical and magnetic resonance imaging findings. BJU Int. 2005;96:373-7. 